\title{
Tuberkulose 2012 - die neuen Empfehlungen zur Therapie der Tuberkulose in Deutschland
}

\author{
Tuberculosis 2012 - Update of Recommendations for the Management \\ of Tuberculosis in Germany
}

Autor

Institut

\section{S. Ewig}

Thoraxzentrum Ruhrgebiet, Kliniken für Pneumologie und Infektiologie, Ev. Krankenhaus Herne und Augusta-Kranken-Anstalt Bochum

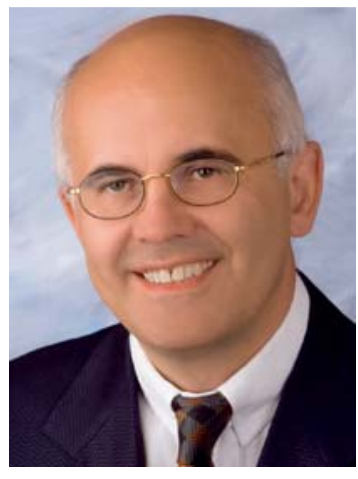

Prof. Dr. med. Santiago Ewig

\section{Bibliografie}

Dol http://dx.doi.org/ $10.1055 / \mathrm{s}-0031-1291876$ Pneumologie 2012; 66: 131-132 (c) Georg Thieme Verlag KG Stuttgart · New York ISSN 0934-8387

\section{Korrespondenzadresse}

Prof. Dr. med. Santiago Ewig

Thoraxzentrum Ruhrgebiet Kliniken für Pneumologie und Infektiologie

Ev. Krankenhaus Herne und Augusta-Kranken-Anstalt Bochum Bergstr. 26

44791 Bochum

ewig@augusta-bochum.de
Aktuelle Empfehlungen zur Therapie der Tuberkulose werden immer wichtiger. Zwar geht die Inzidenz der Tuberkulose in Deutschland weiterhin stetig zurück; während etwa zu Beginn meines Studiums 1978 in der Bundesrepublik noch ca. 30000 Neuerkrankungen pro Jahr erfasst wurden, waren es 2009 im wiedervereinten Deutschland nur noch 4444 Patienten, die an einer Tuberkulose erkrankten. Allerdings nehmen Tuberkuloseerkrankungen durch resistente Stämme und Tuberkulosen bei immunsupprimierten Patienten und bei Patienten mit instabilem sozialem Hintergrund relativ gesehen sogar zu. Parallel dazu nimmt aber auch die Erfahrung in der Behandlung dieser Erkrankung wegen ihrer Seltenheit in Deutschland ab.

In der weltweiten Perspektive ist die Tuberkulose unverändert eine medizinische Herausforderung ersten Ranges, nicht nur wegen ihres epidemischen Charakters, sondern auch wegen der besorgniserregenden Zunahme von Infektionen durch resistente Stämme. In einer globalisierten Welt muss aber auch in Deutschland mit resistenten und multiresistenten Tuberkulosen in der Zukunft vermehrt gerechnet werden.

Nicht zuletzt ist die Tuberkulose angesichts der weltweiten epidemiologischen Situation auch wieder Gegenstand intensiver Forschung, deren Ergebnisse die Behandlungsempfehlungen von heute naturgemäß beeinflussen. In dieser Situation ist die Tuberkulose heute eine Erkrankung, deren Behandlung in die Hände erfahrender und sachkundiger Ärztinnen und Ärzte gehört, und unter diesen sind die Pneumologinnen und Pneumologen besonders gefragt.

Die jetzt vorliegende Aktualisierung der Empfehlungen nimmt Bezug auf internationale Leitlinien und präzisiert diese für die Situation und die Besonderheiten in Deutschland [1]. Dies ist deshalb besonders wichtig, da vor allem die Empfehlungen der WHO der internationalen Situation in ihrer Gesamtheit Rechnung tragen müssen, diese jedoch für Deutschland als Niedrig-Inzidenzland an einigen Stellen modifiziert formuliert werden können bzw. müssen.

Gegenüber der letzten Version der DZK-Empfehlungen sind eine Reihe von wichtigen Modifikationen und Ergänzungen eingeführt worden. Einige wichtige Änderungen sollen hier kurz erwähnt werden.

Einer der bedeutendsten Fortschritte in der Diagnostik der Tuberkulose ist durch die Etablierung der molekulargenetischen Testung auf Resistenzgene erzielt worden. Durch diese Testung ist es möglich geworden, in kürzester Zeit eine Resistenz z.B. gegenüber Rifampicin zu identifizieren, hinter der sich in der Regel eine multiresistente Tuberkulose verbirgt. Die Sensitivität bei Patienten mit mikroskopisch positiver Lungentuberkulose liegt nahe bei $100 \%$ und bei mikroskopisch negativen, kulturpositiven Fällen immerhin noch bei $72-90 \%$ [2]. Ein solcher Test wird nun immer empfohlen, sofern sich anamnestisch (Vortherapie, Kontakt) bzw. epidemiologisch (Herkunft) ein Verdacht auf eine MDR-Tuberkulose ergibt.

Da wir in Deutschland über keine zuverlässigen Daten über eine Ko-Infektion mit dem HI-Virus verfügen, sollte in Zukunft auch jedem Patienten mit Tuberkulose ein HIV-Test angeboten werden. Bei günstigem Therapieausgang sollte eine Röntgen-Thorax-Untersuchung nach 3, 6, 12 und 24 Monaten erfolgen - nicht mehr und nicht weniger Röntgen des Thorax ist erforderlich. Unter den Indikationen für eine stationäre Therapieeinleitung ist die häusliche Situation nun ausdrücklich aufgenommen.

Die Einteilung der Medikamente mit antituberkulöser Wirkung in fünf Klassen wurde von der WHO übernommen. Streptomycin ist nunmehr ein Klasse 2-Medikament in der Gruppe der injizierbaren Medikamente geworden, unter den Klasse 3-Medikamenten (den Fluorchinolonen) werden Ciprofloxacin und Ofloxacin aufgrund verminderter Wirksamkeit nicht mehr empfoh- 
len. Klasse 4-Medikamente umfassen alle Zweitrang-Substanzen mit gesicherter Wirkung gegen M. tuberculosis, Klasse 5-Medikamente diejenigen mit ungesicherter Wirkung.

Zwei Änderungen in den Therapieempfehlungen sind besonders hervorzuheben.

Ethambutol wird nun in der niedrigeren Standarddosierung von $15 \mathrm{mg} / \mathrm{kg} / \mathrm{KG}$ empfohlen (bisher $25 \mathrm{mg} / \mathrm{kgKG}$ ); diese Dosierung reicht für den erwünschten Effekt einer Minderung des Resistenzrisikos aus und vermindert deutlich das Risiko okulärer unerwünschter Wirkungen.

Eine initiale Dreifachtherapie der damals so benannten „Vollsensiblen Minimal-Tuberkulose“ mit Isoniazid, Rifampicin und Pyrazinamid wird jetzt in keiner Indikation mehr für Erwachsene empfohlen. Die Standardtherapie umfasst für die meisten sensiblen Tuberkuloseformen eine initiale Vierfachtherapie über 2 Monate (Isoniazid, Rifampicin, Pyrazinamid und Ethambutol), gefolgt von einer 4-monatigen Kontinuitätsphase mit Isoniazid und Rifampicin. Eine Verlängerung der Therapie auf 9 Monate kann bei ausgedehnter kavernöser Lungentuberkulose oder bei über mehr als 2 Monate persistierend kulturpositiven Verläufen angezeigt sein (dann über 2 Monate als Vierfachtherapie, 1 Monat als Dreifachtherapie (ohne Ethambutol) und sechs Monate als Zweifachtherapie mit Isoniazid und Rifampicin). Eine intermittierende Therapie wird für Deutschland in keiner Indikation empfohlen.

Auf die umfänglichen Empfehlungen für die Therapie von extrathorakalen Tuberkulosen und von Erkrankungen durch mono-, mehrfach- oder multiresistente Stämme kann hier nur hingewiesen werden.

Des Weiteren werden Empfehlungen für die Behandlung von besonderen Patientengruppen (Kinder, HIV-Patienten, Patienten mit Niereninsuffizienz, Patienten mit vorbestehender Leberinsuffizienz, Patienten nach Organtransplantation, Patienten während Schwangerschaft und Stillzeit, Patienten mit der Notwendigkeit einer intensivmedizinischen Behandlung, Patienten mit der Notwendigkeit einer parenteralen Therapie, Patienten mit verminderter enteraler Resorption, Patienten in hohem Lebensalter, Patienten unter TNF-Alpha-Inhibitorentherapie, Patienten mit Siliko-Tuberkulose sowie Patienten nach chirurgischer Resektion eines pulmonalen Tuberkuloms) ebenso gegeben wie für das Management unerwünschter Arzneimittel-Nebenwirkungen und Interaktionen der antituberkulösen Medikamente.

Die Komplexität der Behandlung der Tuberkulose ist trotz abnehmender Prävalenz deutlich gestiegen. Die Formulierung der Standards tritt vor dem Hintergrund nur moderater Evidenz für eine Vielzahl von Empfehlungen zwar nicht mehr mit dem Anspruch einer Richtlinie auf, sondern nur mit dem einer Empfehlung. Dessen ungeachtet ist die Qualität des jetzigen Dokuments gegenüber dem Vorläufer-Dokument [3] deutlich gestiegen. Wir möchten daher den Autoren der Empfehlung, aber auch dem Deutschen Zentralkomitee zur Bekämpfung der Tuberkulose (DZK) unseren Dank und unsere Anerkennung für diese inhaltlich hervorragenden und praktisch äußerst instruktiven Empfehlungen aussprechen. Es sollte uns in der Gesellschaft für Pneumologie und Beatmungsmedizin mit Genugtuung erfüllen, dass aus dem Heimatland Robert Kochs weiterhin international anerkannte Experten hervorgehen, die sich bemühen, ihren Beitrag zur Bekämpfung der Tuberkulose zu leisten.

\section{Literatur}

1 Schaberg T, Bauer T, Castell S et al. Deutsches Zentralkomitee zur Bekämpfung der Tuberkulose (DZK), Deutsche Gesellschaft für Pneumologie und Beatmungsmedizin (DGP). Empfehlungen zur Therapie, Chemoprävention und Chemoprophylaxe der Tuberkulose im Erwachsenen- und Kindesalter. Pneumologie 2012; 66: 131 - 169

2 Boehme CC, Nabeta P, Hillemann D et al. Rapid molecular detection of tuberculosis and rifampin resistance. N Engl JMed 2010; 363: $1005-$ 1015

3 Schaberg T, Forßbohm M, Hauer B et al. Richtlinien zur medikamentösen Behandlung der Tuberkulose im Erwachsenen- und Kindesalter. Pneumologie 2001; 55: 494-511 